\title{
Alternative Wahrnehmungen der Welt gelten lassen?
}

\author{
Herausforderungen eines rassismuskritischen Unterrichts in der Erzieher_innenausbildung
}

Gesellschaftliche Wandlungsprozesse führen zu einer zunehmenden Ungewissheit und einem ProblematischWerden tradierter Wissensformen. Im Zuge des Erstarkens rechtsnationaler Strömungen verschieben sich dabei Grenzen des Sagbaren immer mehr. Wenn aber pädagogisches Handeln immer schon von Offenheit und Ungewissheit, d. h. von Kontingenz, geprägt ist (Paseka et al. 2018) und Wissen prekär zu werden scheint, wie können dann angehende Erzieher_innen in ihrer fachschulischen Ausbildung für das Thema Rassismus sensibilisiert werden?

$\mathrm{D}$ ass hierfür ein spezifisches - rassismuskritisches - Wissen unverzichtbar ist und klare Positionierungen der Lehrkräfte erforderlich sind, zeigen wir anhand von Ausschnitten aus einem Interview mit der Lehrkraft einer Fachschule für Sozialpädagogik. ${ }^{1}$ Differenzsensibles Handeln ist eine Kernaufgabe für Erzieher_innen im Kita-Alltag. Entsprechend spielt die Professionalisierung hierfür auch in den Lehrplänen für die fachschulische Ausbildung in den letzten Jahren

\begin{tabular}{|l|l|} 
Melanie Kuhn \\
Pädagogische Hochschule Heidelberg, Heidelberg, Deutschland \\
*1977; Dr. phil., Professorin für Erziehungswissenschaft mit dem \\
Schwerpunkt Bildung und Ungleichheit an der Pädagogischen \\
Hochschule Heidelberg. Arbeitsschwerpunkte: qualitative, \\
insbesondere ethnographische Kindheits-, Migrations-, \\
Ungleichheits- und Professionsforschung. \\
m.kuhn@ph-heidelberg.de
\end{tabular}

Sandra Landhäußer
Eberhard Karls Universität Tübingen, Tübingen, Deutschland
*1976; Dr. phil., Vertretungsprofessorin am Institut für
Erziehungswissenschaft der Universiät Tübingen. Arbeits-
schwerpunkte: Kinder- und Jugendhilfe, Sozialraum, Beteiligung
in sozialpädagogischen Institutionen und soziale Ungleichheit.
sandra.landhaeusser@uni-tuebingen.de

Zusammenfassung Rassismus kommt als Lerninhalt in den Lehrplänen für die Ausbildung von Erzieher_innen kaum vor. Eine sensible und nicht moralisierende Thematisierung von Rassismus im Unterricht ist so wichtig wie herausfordernd. Die Vermittlung von rassismuskritischem Wissen zum einen und die Selbstpositionierungen der Lehrkräfte zum anderen können dazu beitragen, dass Rassismus nicht unbeabsichtigt bagatellisiert und relativiert wird.

Schlüsselwörter Differenz, Rassismuskritik, Erzieher_innenausbildung, Fachschule eine zunehmende Rolle. Unter sozialen Differenzen werden sozial hergestellte Unterschiede zwischen Individuen oder Gruppen verstanden, die die Lebens- und Bildungschancen von Menschen entscheidend beeinflussen sowie für ihre Identitätsentwicklung und für Diskriminierungserfahrungen bedeutsam sind. Eine zentrale Rolle hierfür spielen die Differenzlinien Klasse/soziale Herkunft, Geschlecht, Rasse/Ethnizität und Behinderung. Differenzsensibel zu handeln bedeutet dann u. a., gesellschaftliche Migrationsrealitäten angemessen zu berücksichtigen. Vor diesem Hintergrund wäre es für die Ausbildung von Erzieher_innen wichtig, dass diese den Schüler_innen Möglichkeiten für eine Auseinandersetzung mit Rassismus bietet und sie zu rassismussensiblem Handeln befähigt.

In rassismuskritischer Perspektive wird Rassismus nicht als individuelles Vorurteil (z. B. von einzelnen Fachkräften) verstanden, sondern als gesellschaftliches Strukturprinzip. Unter dieser Perspektive sind alle gesellschaftlichen Räume, also auch (frühkindliche) Bildungsinstitutionen nicht jenseits, sondern innerhalb gesellschaftlicher Differenz- und Ungleichheitsverhältnisse angesiedelt; es gibt keine Räume außerhalb von Rassismus (Messerschmidt 2016). Vor diesem Hintergrund stellt eine rassismuskritische Professionalisierung eine wichtige Basis für professionelles Handeln auch in Kindertageseinrichtungen dar.

Nimmt man die bundeslandspezifischen Lehrpläne für die Erzieher_innenausbildung in den Blick, dann fällt allerdings auf, dass Rassismus nur in den Lehrplänen von Hessen und Bremen enthalten ist (HE 2015; S. 77; HB 2017, S. 21). Während in Bremen das Thema Rassismus Bestandteil eines verpflichtend $\mathrm{zu}$ absolvierenden Lernfelds ist, wird in Hessen Rassismus im Vertiefungsbereich „Gruppe B, Sozialpädagogische Arbeit im 
interkulturellen Bereich “ zwar benannt, stellt aber kein verpflichtendes Thema für alle Fachschüler_innen dar. Auf dieser Grundlage kann Rassismus als eine weitgehende Leerstelle in den fachschulischen Lehrplänen gelten. Dieser Befund ist nicht isoliert in Bezug auf spezifische Lehrpläne zu sehen, sondern ist eingebettet in die gesellschaftlich stark umkämpfte Frage, ob Rassismus eine angemessene Analysekategorie heutiger gesellschaftlicher Verhältnisse ist (Mecheril und Melter 2010, S. 162). Während Rassismus in Bezug auf die (sozial)pädagogische Praxis und im fachwissenschaftlichen Diskurs zunehmend mehr bearbeitet wird, spiegelt sich die steigende Aufmerksamkeit für Rassismus noch nicht in den Lehrplänen wider.

Demgegenüber finden sich in den Lehrplänen vielfältige Verweise auf Differenz und Inklusion. Inklusion wird dabei als pädagogischer Ansatz präsentiert, mit dem unterschiedliche Differenzen von Adressat_innen angemessen berücksichtigt werden können. Auf einer bundesweiten Ebene bilden im länderübergreifenden Lehrplan (LOAG 2012) das Wahrnehmen und Verstehen von Diversität und die Förderung von Inklusion ein separat ausgewiesenes Lernfeld. Außerdem wird Inklusion als eine von sechs Querschnittsaufgaben formuliert, die quer zu allen Lernfeldern zu berücksichtigen sei. Trotz dieser inklusiven Bezugnahme auf verschiedene Differenzen werden im länderübergreifenden Lehrplan (LOAG 2012) auch immer wieder einzelne Differenzlinien im Sinne einer Aufzählung thematisiert (z. B. Geschlecht, soziale Herkunft, Kultur, Behinderung).

Während im Lehrplan von Baden-Württemberg Differenz als Konstruktion eingeführt wird, erwecken die anderen 15 länderbezogenen Lehrpläne der Fachschulen für Sozialpädagogik den Anschein, als seien Kategorien wie „Kultur“, „Geschlecht“, „Behinderung“ etwas faktisch Gegebenes (Landhäußer und Kuhn 2022). Mit Fokus auf eine Differenzlinie „Kultur“ wird in den meisten Lehrplänen die Annahme vertreten, dass es so etwas wie unterschiedliche „Kulturen“ gibt und sich „,andere Kulturen“ von der „deutschen“ unterscheiden. Verhalten und Handeln von Adressat_innen werden in dieser Perspektive als „kulturbedingt“ erklärt. Diese kulturalistische Deutungsfolie legt nahe, dass Fachkräften Wissen über andere Kulturen vermittelt werden muss, damit sie diese besser verstehen können. Fachwissenschaftlich wurde eine solche Strategie der Interkulturellen Pädagogik umfassend kritisiert, da mit ihr kulturelle Stereotype reproduziert werden (Messerschmidt 2016).

Rassismuskritische Perspektiven tragen dieser Kritik an Interkultureller Pädagogik Rechnung. Sie verwenden Rassismus als einen ,analytische[n] Begriff für die Untersuchung abstammungs- und herkunftsthematisieren- der Ungleichwertigkeitsvorstellungen “ (Messerschmidt 2016, S. 63). Vor diesem Hintergrund wird deutlich, dass hier ein anderer Wissensbegriff zugrunde gelegt wird: Vermittelt wird in einer rassismuskritischen Professionalisierung kein Wissen über die „Anderen“ und deren vermeintlich „kulturbedingten“ Identitäten und Lebensweisen, sondern ein „Wissen über Rassismus“ (Mecheril und Melter 2010, S. 175), das dazu beitragen soll, rassismusrelevante Darstellungs-, Denk- und Handlungsweisen als solche erkennen zu lernen (Fereidooni 2019, S. 3). Die Unterscheidung von „Wir und den Anderen “ wird dabei nicht unreflektiert reproduziert - wie in der Interkulturellen Bildung -, sondern auf ihre gesellschaftliche Funktion hin befragt und dekonstruiert.

\section{Rassismus im Unterricht zum Thema machen}

Da Rassismus in den Lehrplänen kaum vorkommt, stellt sich die Frage, inwiefern Lehrkräfte an Fachschulen dem Thema Rassismus überhaupt eine Bedeutung zuweisen? Nur eine der fünf von uns interviewten Lehrkräfte gibt an, dass Rassismus von ihr als Inhalt des Unterrichts thematisiert wird. Bezeichnenderweise handelt es sich hier um eine Lehrkraft aus Hessen; dem Bundesland, das Rassismus als Unterrichtsthema zumindest im wählbaren Vertiefungsbereich im Lehrplan verankert hat.

Doch welche Bedeutung kann das o.g. rassismuskritische Wissen im Unterricht haben, wenn unter aktuellen gesellschaftlichen Wandlungsprozessen einerseits Wissen brüchiger zu werden scheint und andererseits rechte Positionen und rassistische Diskursmuster hoffähiger werden und den Raum des Sagbaren ausweiten? Gerade auch mit dem Prekär-Werden von Wissen geht eine Lehrer_innenrolle einher, in der diese „keine komfortable, nicht zu gefährdende Position überlegener Einsicht beziehen [können], und es steht - angesichts der massenmedialen Verbreitung - auch kein Hochsitz zur Monopolisierung eines vermeintlich , besseren“ Wissens bereit“ (Paseka et al. 2018, S. 2). Doch dieses BrüchigWerden von „besserem“ Wissen birgt Herausforderungen für eine rassismuskritische Bildungsarbeit. Dies möchten wir am Beispiel des Interviews mit der Lehrkraft zeigen, die als einzige Rassismus als Unterrichtsthema benennt. Im Interview verdeutlicht sie zunächst grundlegende Professionalisierungsziele von differenzbezogenem Unterricht im Allgemeinen:

Und bei der Diversität zum Beispiel, da geht es schon damit los, dass man lernt, dass ein und derselbe Vorgang, den ich sehe in der Welt um mich herum, von anderen ganz anders gesehen wird, dass ich diese Unterschiedlichkeit zunehmend nicht als Bedrohung wahr- 
nehme oder als Hinterfragen meiner Wahrnehmung, sondern gelassen als eine alternative, andere Wabrnehmung der Welt gelten lassen kann. (...) Das ist etwas, was bei Diversität am Anfang steht (...) in meinem Unterricht jetzt ganz einfach, die unterschiedlichen Interpretationen von Vorgängen in der Welt: Ich sehe etwas, was Kinder machen, und zwei Leuten sehen's, würden's ganz anders einschätzen.

Das Thema Diversität führt sie hier zunächst nicht entlang der klassischen Differenzkategorien Klasse/soziale Herkunft, Geschlecht, Rasse/Ethnizität und Behinderung ein, sondern vielmehr bezogen auf eine (Multi) Perspektivität von Erleben und Erfahren (Unterschiedlichkeit von Wahrnehmungen und Interpretationen). Dieses Wahrnehmen und Interpretieren von Vorgängen in der Welt sieht sie bei einzelnen Menschen nicht als graduell, sondern als radikal different (ganz anders). Die Lehrkraft formuliert als grundlegendes Professionalisierungsziel (dass man lernt), dass diese radikale Perspektivität der Wabrnehmung und Interpretation von Geschehnissen und Sachverhalten zunehmend nicht als Bedrohung erlebt, sondern dieser vielmehr mit Akzeptanz begegnet werden solle (gelassen gelten lassen können). Eine solche Haltung, die andere Deutungsperspektiven von Wirklichkeit anerkennt und damit auch der Kontingenz pädagogischer Situationen Rechnung trägt, scheint für sie die notwendige Ausgangsbedingung für einen professionellen Umgang mit Differenz zu sein (was bei Diversität am Anfang steht).

Die starke Betonung, die die befragte Lehrkraft hier auf die Perspektivität und Relativität von Weltwahrnehmung und -interpretation legt, schreibt sich in der Auseinandersetzung mit dem Thema Rassismus im Unterricht fort:

Auch das ist (...) für mich eine ganz wichtige Sache in meiner Lehrerrolle; alle diejenigen, die sagen "Also ich sehe hier keinen Rassismus" - und dann denke ich mir ja, und jetzt kann ich anfangen als Lehrer zu sagen: „Und du musst jetzt Rassismus sehen. “ Und dann hab ich sozusagen den größten Fehler gemacht, den ich machen kann. Ich muss des dann auch stehen lassen können bestimmte Dinge, die den Unterschied zu mir darstellen.

Die Lehrkraft beschreibt hier beispielhaft, was für sie ein zentrales Prinzip (wichtige Sache für meine Lehrerrolle) ist, wenn Rassismus im Unterricht zum Thema wird. Wenn manche Schüler_innen (alle diejenigen) einen Sachverhalt im Unterricht als nicht rassistisch benennen (keinen Rassismus sehen), dann gilt es für sie, diese Deutung ihrer Schüler_innen zu akzeptieren (steben lassen können); auch dann, wenn sie selbst eine andere Einschätzung zur Rassismusrelevanz eines Geschehens hat (Dinge, die den Unterschied zu mir darstellen). In einer drastischen Beschreibung weist die Lehrkraft als ein worst-case-Szenarium einer rassismuskritischen Professionalisierung aus (den größten Fehler), den Schüler_innen die eigene Deutungsperspektive einer Situation aufzuoktroyieren (du musst Rassismus sehen). Die in der ersten Sequenz beschriebene Einübung der Schüler_innen in die Fähigkeit zur Achtung und Akzeptanz aller Perspektiven des Gegenübers ist also nicht nur Professionalisierungsziel, sondern zugleich ein grundlegendes Prinzip der Unterrichtsgestaltung; im Verlauf des Interviews betont sie immer wieder das Anliegen, den eigenen Unterricht möglichst hierarchiefrei und in partizipativer Abstimmung mit den Schüler_innen auszugestalten (Kuhn 2022). Deutlich wird, dass die Lehrkraft ihre eigene Perspektive auf Rassismus nicht monopolisiert und als ein „besseres“, den Schüler_innen überlegenes Wissen ausweist. Dies ließe sich als eine Unterrichtsgestaltung verstehen, die demokratieorientiert und wenig moralisierend die Perspektiven und Weltsichten der Schüler_innen respektiert. Dies kann dazu beitragen, dass Schüler_innen sich trauen, ihre Positionen und Ansichten im Klassenraum offen zu äußern. Denn Moralisierungen und Skandalsierungen in der Thematisierung von Rassismus können Lernchancen eher verhindern als ermöglichen (Fereidooni 2019, S. 8).

\section{Gefahr der Relativierung von Rassismus}

Wenn die befragte Lehrkraft allerdings beim bloßen stehen lassen können der formulierten Schüler_innenposition, dass etwas kein Rassismus sei, verbleibt, ${ }^{2}$ und dies nicht zum Gegenstand weiterer Auseinandersetzungen macht, dann ist zu fragen, welche sozialen Effekte dieses stehen lassen können in der Unterrichtssituation haben kann?

Wenn unterschiedliche Einordnungen von Geschehnissen als (nicht)rassistisch nebeneinander stehen bleiben, dann wird Rassismus implizit zu einem beliebigen, deutungsoffenen, perspektiven- und meinungsabhängigen Phänomen. Dies kann unbeabsichtigt einen Beitrag dazu leisten, dass Rassismus relativiert, bagatellisiert und auf eine subjektive „Ansichtssache“ reduziert wird, die ausschließlich im Auge der Betrachter_innen liege. Rassismuskritische Bildungsarbeit erfordert zu reflektieren, wie Lehrende und wie Schüler_innen innerhalb gesellschaftlicher Ungleichheitsverhältnisse positioniert sind, wer von welchem Standort aus spricht und welche Effekte das Sprechen auf die Schüler_innen hat (Messerschmidt 2016, S. 62). Was kann das stehen lassen können für 
Schüler_innen der Klasse bedeuten, die möglicherweise selbst faktisch oder potenziell von Rassismuserfahrungen betroffen sind? Diese erleben Unterrichtssituationen, in denen Rassismus abgesprochen, kleingeredet oder banalisiert wird, mitunter als eine Re-Viktimisierung und als sekundäre Rassismuserfahrung (Çiçek et al. 2014), weil damit auch eigene Rassismuserfahrungen in Gefahr stehen, nicht ernst genommen zu werden.

Rassismuskritische Bildungsarbeit in schulischen Kontexten erfordert nicht nur, dass Schüler_innen lernen, rassismusrelevante Sachverhalte zu „erkennen“ und auch „menschenfeindliche Positionen “ zu benennen (Fereidooni 2019, S. 3), sie muss zugleich mitbedenken, dass Schüler_innen zwar alle, aber durch ihre Vorerfahrungen und Lebensrealitäten eben unterschiedlich, in Rassismus verstrickt sind. Diese spezifischen Betroffenheiten und Verletzlichkeiten gilt es mit zu berücksichtigen. Dieses Geschehen ist allerdings nicht nur für die Unterrichtssituation und die Fachschüler_innen selbst folgenreich. Wird berücksichtigt, dass hier angehende Erzieher_innen für ein zukünftiges differenzsensibles Handeln in Kindertageseinrichtungen ausgebildet werden, dann ist dieses stehen lassen in professionalisierungsbezogener Hinsicht durchaus nicht unproblematisch.

\section{Rassismuskritische Bildungsprozesse initiieren}

Wie können rassismuskritische Bildungsprozesse im Unterricht durch die Benennung und die Bearbeitung rassismusrelevanter Geschehnisse angeregt werden? Welche Bedeutung hat Wissen - trotz oder gerade unter der Annahme des Prekär-Werdens von Wissen - dabei? Fereidooni (2019, S. 8) problematisiert hier das Ignorieren genauso wie das Skandalisieren und Moralisieren, das Schüler_innen als Rassist_innen entlarvt. Erforderlich ist vielmehr eine nicht moralisierende und nicht skandalisierende Thematisierung rassismusrelevanter Geschehnisse, wie sie eine rassismuskritische Perspektive anstrebt (ebd.).

Wenn eine solche Perspektive gerade nicht voraussetzt, dass jede Unterscheidung und jede Form der Diskriminierung von Migrationsanderen rassistisch ist (Mecheril und Melter 2010, S. 168), dann liegt der Ausgangspunkt für eine solche sensible Thematisierung darin, Fachschüler_innen ein rassismuskritisches Wissen zu vermitteln, mit dem sie erst befähigt werden, Rassismus als Rassismus erkennen zu können. Es braucht z. B. geschichtliches Wissen, da abwertende Bedeutungen von Begrifflichkeiten oft in historischen Zusammenhängen entstanden sind und tradiert werden (vgl. u. a. Mecheril und Melter 2010, S. 175f.). Eine gemeinsame Auseinandersetzung würde nicht dabei stehen bleiben, ein Geschehen entweder als rassistisch oder nicht rassistisch einzuordnen. Es wäre mit den Schüler_innen darüber in einen differenzierenden Diskurs zu treten, inwiefern, vor dem Hintergrund welcher Begriffstraditionen und historischer Ereignisse, „in welcher Weise, unter welchen Bedingungen“ Sachverhalte oder beispielsweise Handlungspraktiken und nicht zuletzt Selbstverständnisse einzelner Personen, Gruppen oder auch gesellschaftlicher Institutionen „durch Rassismen vermittelt sind" (Mecheril und Melter 2010, S. 172). Dabei wären ebenfalls die möglichen Uneindeutigkeiten, Ambivalenzen, Brüche und Verschiebungen rassismusrelevanter Begriffe, Diskurse und Handlungspraxen offensiv zu thematisieren. Darüber hinaus müsste es immer auch um die Konsequenzen gehen, die rassismusrelevante Konstruktionen und insbesondere auch Rassismus verneinende, bagatellisierende und relativierende Diskurse für betroffene Subjekte und für gesellschaftliche (De) Privilegierungs- und Hierarchisierungsprozesse haben. Diese diskursorientierte Praxis zielt weder auf Schuldzuweisungen noch darauf, Schüler_innen zu zwingen, Rassismus zu sehen, sondern vielmehr darauf, sie zu ermutigen, „ihre eigenen Wissensbestände aus einer rassismuskritischen Perspektive [zu] reflektieren“ (Simon und Fereidooni 2020, S. 4). Eine Selbstpositionierung der Lehrkraft zum Thema Rassismus, mit der sie transparent macht, warum sie selbst ein Geschehen unter welchen Kriterien und auf der Basis welchen Wissens als rassistisch einschätzt, würde nicht nur dazu beitragen, dass die Schüler_innen dazu angeregt werden, sich zu Rassismus zu positionieren und im Sinne der Kinder in ihrer späteren Berufspraxis zu intervenieren. Sie hätte auch den Effekt, die Verneinung von Rassismus zwar als eine Perspektive gelten zu lassen, aber eben gerade nicht unkommentiert stehen zu lassen.

Eingegangen. 28. September 2021

Angenommen. 22. November 2021

Funding. Open Access funding enabled and organized by Projekt DEAL.

Open Access. Dieser Artikel wird unter der Creative Commons Namensnennung 4.0 International Lizenz veröffentlicht, welche die Nutzung, Vervielfältigung, Bearbeitung, Verbreitung und Wiedergabe in jeglichem Medium und Format erlaubt, sofern Sie den/die ursprünglichen Autor(en) und die Quelle ordnungsgemäß nennen, einen Link zur Creative Commons Lizenz beifügen und angeben, ob Änderungen vorgenommen wurden.

Die in diesem Artikel enthaltenen Bilder und sonstiges Drittmaterial unterliegen ebenfalls der genannten Creative Commons Lizenz, sofern sich aus der Abbildungslegende nichts anderes ergibt. Sofern das betreffende Material nicht unter der genannten Creative Commons Lizenz steht und die betreffende Handlung nicht nach gesetzlichen Vorschriften erlaubt ist, ist für die oben aufgeführten 
Weiterverwendungen des Materials die Einwilligung des jeweiligen Rechteinhabers einzuholen.

Weitere Details zur Lizenz entnehmen Sie bitte der Lizenzinformation auf http://creativecommons.org/licenses/by/4.0/deed.de.

1. In der Studie „Differenz und Ungleichheit in der fachschulischen Ausbildung von Erzieher_innen (DUfA) " haben wir Dokumentenanalysen der fachschulischen Curricula für die Erzieher_innenausbildung (Landhäußer und Kuhn 2022) sowie bislang fünf Expert_ inneninterviews mit Lehr- und Leitungskräften aus Fachschulen für Sozialpädagogik in Baden-Württemberg (BW), Rheinland-Pfalz (RP) und Hessen (HE) geführt.

2. Die Rede vom stehen lassen können deutet zumindest darauf hin, dass diese Rassismus de-thematisierenden Deutungsweisen von Schüler_innen im Unterricht unkommentiert stehen bleiben könnten. Ob die befragte Person sich im Unterricht selbstpositioniert und den Schüler_innen gegenüber transparent macht, dass und warum sie selbst einen Sachverhalt für rassismusrelevant hält, muss hier letztlich offenbleiben. Zumindest spricht sie dies in dieser Interviewsequenz nicht an.

\section{Literatur}

Fereidooni, K. (2019). Rassismuskritik für Lehrer_innen und Peers im Bildungsbereich. Berlin: Schwarzkopf-Stiftung Junges Europa.

HB (2017). Die Berufsbildenden Schulen im Land Bremen. https://www. ibs-bremen.de/wp-content/uploads/2020/07/rahmenplan-fachschule-fuersozialpaedagogik.pdf (Erstellt: 06.2017). Zugegriffen: 15. Nov. 2021. Sozialpädagogik. Rahmenplan für die Fachschule.

HE (2015). Lehrplan für die Ausbildung von Erzieherinnen und Erziehern an der Fachschule für Sozialwesen, Fachrichtung Sozialpädagogik. Wiesbaden: Hessisches Kultusministerium. Entwurf. Version 13, Stand: 14.01.2015

Kuhn, M. (2022). Von widersprüchlichen Verhältnissen, strukturellen Begrenzungen und ausgeloteten Spielräumen: Partizipation in der fachschulischen Erzieher_innenausbildung. In S. Benedetti, N. Brück, A. Kirchner, N. Köbel \& O. Rollmann (Hrsg.), Moral-Menschenrechte - Demokratie. Wiesbaden: Springer VS. im Erscheinen.

Landhäußer, S., \& Kuhn, M. (2022). Differenz in der fachschulischen Ausbildung frühpädagogischer Fachkräfte. In M. Bader, S. Dahlheimer \& S. Geiger (Hrsg.), Perspektiven auf Heterogenität in Bildung und Erziehung. Kindheits- und Sozialpädagogische Beiträge. Weinheim: Beltz Juventa. im Erscheinen.

LOAG (2012). Kompetenzorientierter länderübergreifender Lehrplan für die Ausbildung von Erzieherinnen und Erziehern. https://www.boefae.de/ wp-content/uploads/2012/11/laenderuebergr-Lehrplan-Endversion.pdf (Erstellt: 1. Juli 2012). Zugegriffen: 10. Okt. 2021.

Mecheril, P., \& Melter, C. (2010). Gewöhnliche Unterscheidungen. In P. Mecheril, A. Kalpaka, C. Melter, İ. Dirim \& M. do Mar Castro Varela (Hrsg.), Migrationspädagogik (S. 151-198). Weinheim, Basel: Beltz.

Messerschmidt, A. (2016). Involviert in Machtverhältnisse. In A. Doğmuş, Y. Karakaşoğlu-Aydın \& P. Mecheril (Hrsg.), Pädagogisches Können in der Migrationsgesellschaft (S. 59-70). Wiesbaden: Springer VS.

Paseka, A., Keller-Schneider, M., \& Combe, A. (2018). Einleitung. In A. Paseka, M. Keller-Schneider \& A. Combe (Hrsg.), Ungewissheit als Herausforderung für pädagogisches Handeln (S. 1-12). Wiesbaden: Springer VS.

Simon, N., \& Fereidooni, K. (2020). Rassismus(kritik) und Fachdidaktiken - (K)ein Zusammenhang? In N. Simon \& K. Fereidooni (Hrsg.), Rassismuskritische Fachdidaktiken. Theoretische Reflexionen und fachdidaktische Entwürfe rassismuskritischer Unterrichtsplanung (S. 1-17). Wiesbaden: Springer.

Ciçek, A., Heinemann, A., \& Mecheril, P. (2014). Warum die Rede, die direkt oder indirekt rassistische Unterscheidungen aufruft, verletzen kann. In G. Hentges, K. Nottbohm, M. M. Jansen \& J. Adamou (Hrsg.), Sprache Macht-Rassismus (S. 309-326). Berlin: Metropol.

Hier steht eine Anzeige. Springer 